\title{
Journal Bearing Fault Detection Based on Daubechies Wavelet
}

\author{
Narendiranath Babu T. ${ }^{(1)}$, Himamshu H.S. ${ }^{(1)}$, Prabin Kumar N. ${ }^{(1)}$ \\ Rama Prabha D. ${ }^{(2)}$, Nishant C. ${ }^{(1)}$ \\ (1) School of Mechanical Engineering, VIT University \\ Vellore, Tamil Nadu, India; e-mail: narendiranathbabu.t@vit.ac.in \\ (2) School of Electrical Engineering, VIT University \\ Vellore, Tamil Nadu, India \\ (received October 17, 2016; accepted April 12, 2017)
}

\begin{abstract}
Journal bearings are widely used to support the shafts in industrial machinery involving heavy loads, such as compressors, turbines and centrifugal pumps. The major problem that could arise in journal bearings is catastrophic failure due to corrosion or erosion and fatigue, which results in economic loss and creates major safety risks. Thus, it is necessary to provide suitable condition monitoring technique to detect and diagnose failures, and achieve cost savings to the industry. Therefore, this paper focuses on fault diagnosis on journal bearing using Debauchies Wavelet-02 (DB-02). Nowadays, wavelet transformation is one of the most popular technique of the time-frequency-transformations. An experimental setup was used to diagnose the faults in the journal bearing. The accelerometer is used to collect vibration data, from the journal bearing in the form of time domain. This was then used as input for a MATLAB code that could plot the time domain signal. This signal was then decomposed based on the wavelet transform. The fast Fourier transform is then used to obtain the frequency domain, which gives us the frequency having the highest amplitude. To diagnose the faults various operating conditions are used in the journal bearing such as Full oil, half loose, half oil, fault 1, fault 2, fault 3 and full loose. Then the Artificial Neural Networks (ANN) is used to classify faults. The network is trained based on data already collected and then it is tested based on random data points. ANN was able to classify the faults with the classification rate of $85.7 \%$. Thus, the test process for unseen vibration data of the trained ANN combined with ideal output target values indicates high success rate for automated bearing fault detection.
\end{abstract}

Keywords: journal bearing; fault diagnosis; Debauchies wavelet; artificial neural network.

\section{Introduction}

When there is too much vibration due to faults in the journal bearing, the abnormal change in the vibration can be detected using an accelerometer which would be more advantageous. Frequency and amplitude are the two important parameters which can be used to detect failure of the bearings at an early stage. Apart from the visual testing, the chance for prognostics must be coupled with new monitoring techniques such as vibration monitoring, which could indicate problems within the bearing assembly as early as possible (NAREndiranath BABU T. et al., 2015).

Several new parameters are deduced to express the synthesized manufacturing tolerances and the structural parameters of the journal bearing system based on a new dimensionless modified Somerfield number.
The work also shows that the journal bearing system is insensitive to the manufacturing tolerances when the system eccentricity ratio runs around $0.3-0.4$ (XU et al., 2009). The fault diagnosis system of Tunnel Boring Machine (TBM) is developed by Lab VIEW and Matlab, and the operation result proves the validity of the algorithm and the feasibility of the condition monitoring technique (TIANRUi et al., 2013).

Continuous wavelet transforms (CWTs) are widely recognized as effective tools for vibration-based machine fault diagnosis (TsE et al., 2004), as CWTs can detect both stationary and transitory signals. However, due to the problem of overlapping, a large amount of redundant information exists in the results that are generated by CWTs. The appearance of overlapping can smear the spectral features and make the results very difficult to interpret for machine opera- 
tors. KAREEM (2015) concluded that the failure of crankshafts in automobile is largely caused by oil leakages in engines, overloading, misalignment, poor surface finish, poor reconditioning of thrust bearing and adulterated engine oil. Filter design aspects of a frequency scanning receiver have been presented covering pre selection of sub-bands, intermediate frequency filtering for image rejection. Also, digital filters are used to reduce the additional noise generated due to electrical interference during running conditions of the motor. This filter is used to minimize the error between the idealized filter characteristics and the actual filter range (DEHM-ANDONE et al., 2012).

Failure analysis investigations were carried out on a failed interfering part of journal bearing related to turbine of a steam power plant. In order to indicate the failure mechanism, visual and experimental investigations were performed. The service life of journal bearings can be improved by using appropriate assembly with required clearance (MEHDIZADEH, KHODABAKHSHI, 2014; Feng et al., 2011). Hydrodynamic bearings (HBs) are prone to initial wear due to contact friction between the bushing and shaft under the condition of insufficient hydrodynamic pressure (IHP).

FENG et al. (2011) studied the working principle of rolling contact bearing and the frequent faults of the bearing occurred to overheating and misalignment of shafts. Then the faults are induced in the bearing and the vibrational analysis is done and readings recorded. A pattern was then obtained using this data and hence stated as a way to detect these faults without having to stop the machinery. The quality teams in industries face an uphill task to determine the lifetime of the journal bearing component of any machinery. Some high accuracy machinery requires regular checks to determine the margin of error and compare with the allowable range. Hence, there is a need of experimental setup to diagnose the faults. In the investigation, the ARLW filter is used to extract the most fault-related components in the vibration signal, namely impulsive features. The Antisymmetric Real Laplace Wavelet (ARLW) filter is optimized by Differential Evolution DE using the kurtosis maximization criterion.

WANG and Chen (2011) proposed an intelligent diagnosis method for detection of the bearing faults on the basis of possibility theory and a fuzzy neural network in that analysis was done on the vibration features extracted from frequency domain signal.

HAKIM and RAZAK (2014) discussed that ANNs are vastly being used in both industries and modern research labs for essential purposes. Some of ANNs' common applications are pattern recognition, chemical compound identification, process control, industrial temperature and force prediction, stock market prediction, making video games intelligence, voice recognition. Apart from these, ANNs have already been rep- resented as a decent method for monitoring and resolving several structural health-related problems' faults in mechanical systems' and heat conduction based on inverse problems.

The wavelet transform resolves all the deficiencies such as bearing faults, gear faults and provides good frequency resolution and low time resolution for lowfrequency components as well as low frequency resolution and good time resolution for high-frequency components. Therefore, the wavelet transform has been widely applied in the field of vibration signal analysis and feature extraction for bearing (KANKAR et al., 2011).

In the case of the feature extraction, authors come across a wide range of classifiers used for fault diagnostics in bearings. The classifiers based on artificial neural networks (LAZZERINI, VOLPI, 2013) demonstrated a highly reliable classification.

RAFIEE et al. (2010) have developed a procedure which experimentally recognizes gears and bearings faults of a typical gearbox system using a multi-layer perceptron neural network. They have further proposed a technique for selecting mother wavelet function using an intelligent fault diagnosis system. The type of gear failures of a complex gearbox system is identified using genetic algorithm and artificial neural networks. They have shown that the Daubechies wavelet is the most effective for both faulty gears and bearings.

HARIHARAN and SRINIVASAN (2012) implemented radial basis function network and probabilistic neural network for the classification of rolling element bearing faults. A combination of signal's time varying statistical parameters and characteristic rolling element bearing fault frequency components, obtained through the frequency spectrum analysis method, were used as the extracted features of each recorded vibration signal. The classification results showed whether the bearing was normal or defective using neural network.

According to the non-stationary and nonlinear characteristics of REB vibration signals, feature extraction method is based on empirical mode decomposition (EMD) energy entropy in ALI et al. (2015). Therefore, the chosen features are used to train an artificial neural network (ANN) to classify bearings defects. Experimental results indicated that the proposed method based on run-to-failure vibration signals can reliably categorize bearing defects. It shows potential application of ANN as effective tool for automatic bearing performance degradation assessment without human intervention.

The vibration sensors used on wavelet technique are basically accelerometers. The sensor characteristics, the pre and post-processing, and the analysis methods for tower, main bearing and planet gears are different from the high speed drive train and generator due to low and high frequency ranges, respectively. In addition, these different frequency ranges add more chal- 
lenges to wind turbine monitoring, mainly due to cross frequencies and low frequency components, which are harder to monitor. Both time and frequency domain signals can be used to diagnose faults. However, it is possible to extract more information from these signals through signal processing algorithms and alarms such as envelope signal and narrow band envelope alarms. Furthermore, there are several statistical condition indicators such as root mean square (RMS), crest factor, peak-to-peak, kurtosis and sidebands factors that can be used to extract useful information from measured vibration signals (GUO, INFIELD, 2012).

Another way to monitor the vibration level is through wavelet transformations. It has been successfully applied to misalignment and bearing faults. In addition, it can be used as a general indication of a fault produced in a wind turbine (LIU et al., 2012). Wavelet analysis is based on a set of functions called daughter wavelets, which are obtained through time translation and amplitude dilation of a mother wavelet. Wavelet can map any signals in such a way that it is possible to have time-frequency representation of the signal. Therefore, it can be used to process non-stationary data and it is capable of quantifying/identifying the location and level of damage.

However, vast quantities of the failures are caused due to a bearing failure. Therefore, bearing condition monitoring becomes crucial. Author aims at providing a state of the art review on wind turbine bearing condition monitoring techniques such as acoustic measurement (DE AzEvEDo et. al., 2016).

DAzBrowski and DzIURDź (2016) presented the proposition of algorithm of noise minimization at the machine operator's stand. To do this they had used coherent analysis allowing for identification of paths of vibroacoustic energy propagation.

Chen et. al. (2016) reviewed Wavelet Transform (WT) to rotating machinery fault diagnosis. Inner product essence validation of WT in rotating machinery fault diagnosis (RMFD) is firstly carried out by simulation and field test experiments. Then the development process of WT based on inner product is concluded and the corresponding applications in RMFD are summarized.

WANG and Chen (2011) proposed sequential diagnosis technique through which the fuzzy neural network realized by the partially-linearized neural network (PNN) can sequentially identify fault types. Possibility theory and the Mycin certainty factor are used to process the ambiguous relationship between symptoms and fault types.

Hence, this experimental research highlights the importance of vibration monitoring to diagnose the faults on the journal bearing caused by high rotational speeds. This is the first known attempt in fault diagnosis on journal bearing in rotating machines. It has been concluded, that vibration parameters such as am- plitude can be used as a reliable and sensitive tool for the early detection of faults between the surfaces of a bearing and high speed shaft. With reasonable approaches, this model will allow us to interpret the result of fault units, as well as predict the behaviour of rotating machines and the features of tribology pairs in industrial experiments. Lastly, a new approach was proposed for automatic classification of bearing conditions through Artificial Neural Network. The Artificial Neural Network (ANN) was created, trained and tested using Neural Network Toolbox with LevenbergMarquarat Back Propagation (LMBP) training Algorithm. The Neural Network test process for unseen vibration data of the trained ANN combined with the ideal output target values has been presented.

\section{Wavelet transform}

A wavelet series is a representation of a squareintegrable (real- or complex-valued) function by a certain orthonormal series generated by a wavelet. The wavelet transform is used to construct time-frequency representation of the signals. A wavelet can be described as a small wave which oscillates and decays in the time domain. Wavelet transform consists of two functions: scaling function which has the scaling index $k$ and the time translation function with time translation index $j$. Among the different transform types the Daubechies transform is found appropriate in denoising. Denoising consists of mainly three steps: decomposition, thresholding and signal reconstruction. In decomposition, the noise signals are decomposed to four levels using Daubechies wavelet (DB02). In thresholding, we use soft thresholding. The soft thresholding zeroes out all the values smaller than the threshold value and subtracts the threshold from the values greater than the threshold. Let the wavelet coefficients are represented as $T_{j, k}$. If the threshold can be represented as $\mathrm{t}$, then the thresholding can be represented as,

$$
\begin{aligned}
D\left(T_{j, k}\right)=1 & \text { for } \quad T_{j, k} \geq t, \\
0, & \text { otherwise. }
\end{aligned}
$$

Figure 1 shows a Daubechies wavelet, which is a function in contrast to sine/cosine functions used in the Fourier analysis which is localized in time PERCI-

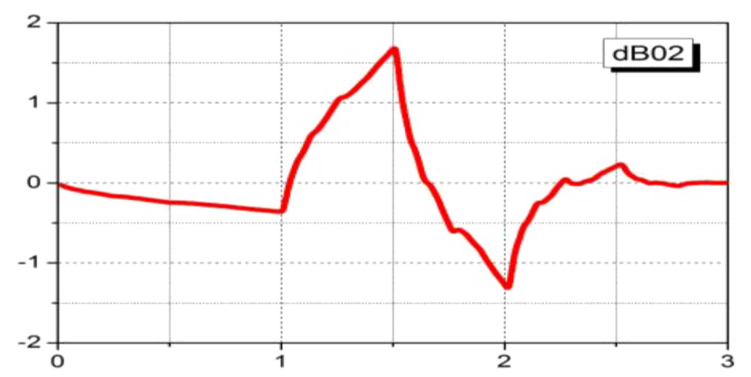

Fig. 1. Daubechies wavelet. 
VAL, Walden (2000) and Walnut (2004). It is much better suited to analysis of the signals that are not periodical, such as for instance pulsed noise signals (Piotrowski et al., 2010).

To overcome the mismatch between uniform filter banks and spectral decomposition of the journal bearing, a non-uniform filter bank was proposed for the bearing model. A traditional journal bearing model is used, and to overcome the above-mentioned problem, the Daubechies wavelet (DWT) DB02 was used. This is the only non-uniform FIR filter bank that combines perfect signal reconstruction with energy conservation and removes the aliasing effect. In DWT analysis, filters are used with different cut-off frequencies at different scales (SRINivasan, JAMIESON, 2009). DWT offers a compact representation of the signal in the time and frequency domains along with efficient computation in the form of Eqs. (3) and (4).

$$
\begin{aligned}
d_{j k} & =\int x(t) \mathrm{d} t \\
& =2^{j / 2} \int x(t) \emptyset_{j k}\left(2^{j} t-k\right) \mathrm{d} t, \\
\emptyset_{j k}(t) & =2^{j / 2} \emptyset_{j k}\left(2^{-j} t-k\right) \mathrm{d} d, \quad j, k \in Z, \\
\int x^{k} \psi(x) \mathrm{d} x & =0, \quad \text { for } \quad 0 \leq k \leq K,
\end{aligned}
$$

where $d_{j k}$ is the wavelet coefficient and $x(t)$ is the time signal. The number of vanishing moments is related to the smoothness and flat frequency response of wavelet filters. A large number of vanishing moments produces a more compact signal. A vanishing moment must satisfy the condition given in Eq. (5), where $K$ is the degree of the polynomial ( 0 to $K)$. However, the length of filter increases with the number of vanishing moments at the cost of complexity and time for computation (Srinivasan, Jamieson, 1998).

\section{Experimental setup and procedure}

A mechanical system was designed to operate the journal bearing. The experimental system consists of a three phase AC driving motor, couplings, load setup, a ball bearing, a journal bearing with the main drive as the shaft. As shown in Fig. 2 all these components are installed on a base frame. The load setup is placed at one end of the test rig. The electric drive motor is connected to the main shaft which is coupled with a hard rubber.

The journal bearing is mounted at the right end of the drive train. The journal bearing is shown in Fig. 2. In order to maintain the axial displacement of the journal bearing, a pair of washers is installed on the fasteners. The washer acts as a stopper to prevent axial displacement and it also serves as a thrust bearing. The preliminary experimental results showed that

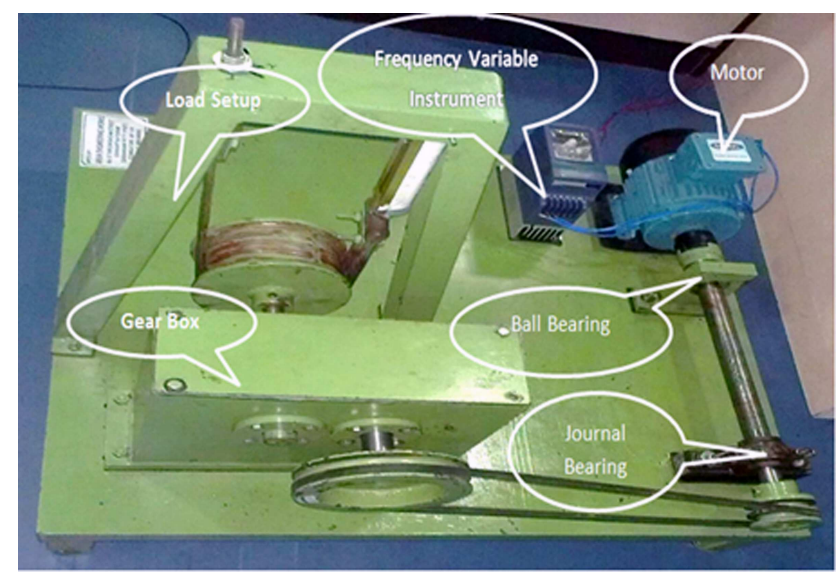

Fig. 2. Experimental setup and faults on the surface of the bearing.

the high radial loading of the journal bearings generate a high temperature and to reduce the temperature, a rolling bearing is placed between the motor and the journal bearing. Table 1 shows the dimensions of the journal bearing made of gun metal.

Table 1. Dimensions of the journal bearing made of gun metal.

\begin{tabular}{|l|c|}
\hline Bore diameter $[\mathrm{mm}]$ & 25.5 \\
\hline Length $[\mathrm{mm}]$ & 50.51 \\
\hline Weight $[\mathrm{kg}]$ & 0.248 \\
\hline Maximum load $[\mathrm{N}]$ & 175 \\
\hline Maximum speed $[\mathrm{rpm}]$ & 3700 \\
\hline
\end{tabular}

The experimental setup consists of a motor of $1.5 \mathrm{HP}, 3700 \mathrm{rpm}$ connected to the rotor, through a coupling supported with one ball bearing and a journal bearing at the end of pulley which is attached to a loading system as shown in Fig. 2. The vibration data is collected from the journal bearing using an accelerometer (621B40) and the vibration signals are processed with DEWESOFT software. Peaks of low amplitude occur when bearing is normal, but amplitude of vibration is bigger when there are defects in the journal bearing. The maximum amplitudes of vibration in the power spectrum plot indicates that there is a fault on the bearing.

In the experimental investigation, the vibration signals were collected at an ideal condition without any crack or wear, i.e. healthy surface at a shaft speed of $1240 \mathrm{rpm}$. The readings were recorded at a sampling rate of $12000 \mathrm{~Hz}$. The 621B40 M I accelerometer type, sensor having sensitivity $1.02 \mathrm{mV} /\left(\mathrm{m} \cdot \mathrm{s}^{2}\right)$ and frequency range up to $12 \mathrm{kHz}$, is used in horizontal position on the bearing housing with magnetic base to collect the vibration data. The other end of the accelerometer is connected to the vibration data collector with DWESOFT software. Finally, MATLAB software is used for various signal processing. 


\section{Seeded faults and signal processing}

Mostly, faults in the journal bearing are caused by the presence of impurities in the lubricants used. Solid contaminants caused a sudden damage to the bearing surface. Due to the presence of contaminants in the oil, there was a change in the viscosity. This led to a deterioration of the surface and caused excessive vibration culminating in a fault. This was found after a series of experiments.

\subsection{Faults in the journal bearing surface}

In journal bearing, the failure often occurs due to scratching. A healthy bearing surface (without fault) is shown in Fig. 3a. An artificial fault is made by making cuts circumferentially on the inner surface of the bearing, every $2 \mathrm{~mm}$, along the length of the bearing using a lathe machine. a)

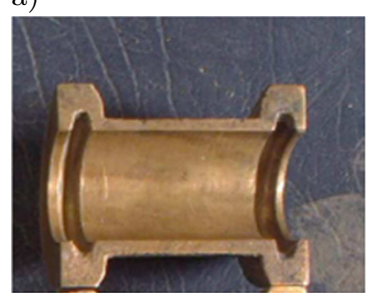

c)

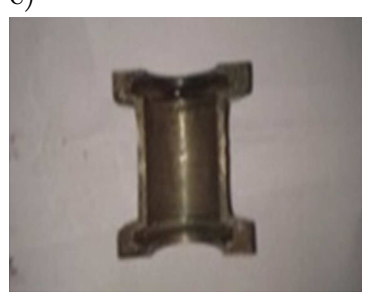

b)

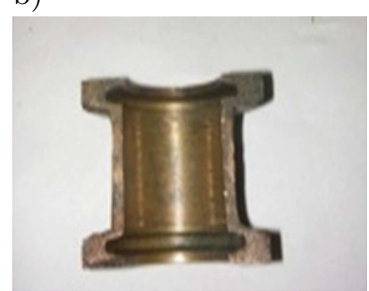

d)

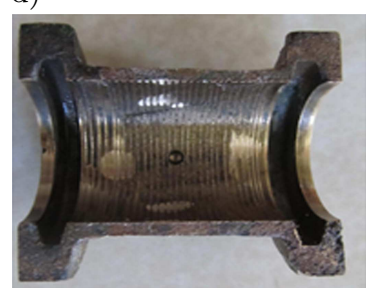

e)

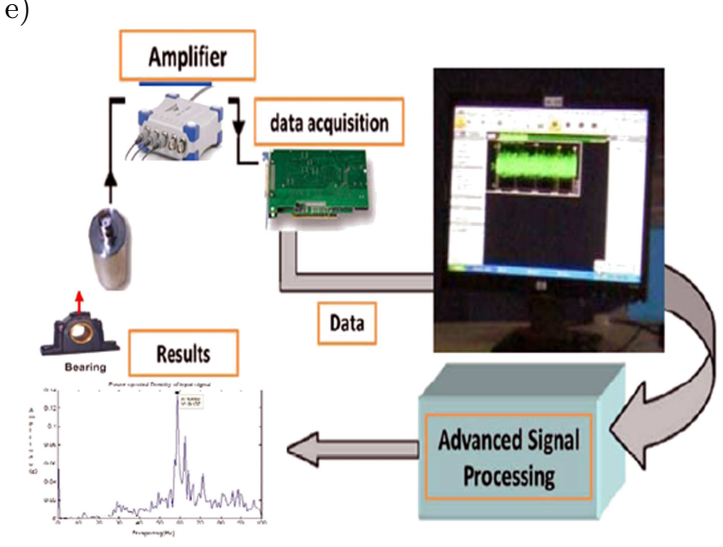

Fig. 3. Faults in the journal bearing surface and layout of signal processing unit: a) no fault on the journal bearing, b) bore fault on the bearing (Fault F1), c) bore fault with hole on the bearing (Fault F2), d) material removal and bore fault with hole on the bearing (Fault F3), e) layout of the signal processing unit through vibration data acquisition system.
The journal bearing with the eccentric bore fault of $0.07 \mathrm{~mm}$ depth and $0.2 \mathrm{~mm}$ width is shown in Fig. 3b. Then, the second fault is made by drilling a hole of $3 \mathrm{~mm}$ diameter and $2 \mathrm{~mm}$ depth as shown in Fig. 3c. A third random fault is made using a grinding wheel to remove the material from the inner surface of the bearing which is shown in Fig. 3d.

The fault on the bearing surface leads to changes in the local roughness on the surface of a journal bearing. This in turn leads to change in the distribution and size of the local asperities which can enhance and cause changes in amplitude and frequency of vibration generated from the bearing surface.

The seven different states of the journal bearing are chosen based on best judgment. These states would have to be validated to check if unique patterns can be obtained from the same and hence demand a classification of its own. These are as shown in Table 2 . The faults were introduced manually using grinding machines and drilling machines.

Table 2. List of faults induced.

\begin{tabular}{|c|c|c|}
\hline $\begin{array}{c}\text { Serial } \\
\text { Number }\end{array}$ & Fault & Description \\
\hline 1 & $\begin{array}{l}\text { Healthy } \\
\text { bearing } \\
\text { (full oil) }\end{array}$ & $\begin{array}{l}\text { This represents the good condi- } \\
\text { tion bearing properly fixed and } \\
\text { with continuous oil supply. }\end{array}$ \\
\hline 2 & Half loose & $\begin{array}{l}\text { The screws holding the bearings } \\
\text { are slightly loosened. }\end{array}$ \\
\hline 3 & Half oil & $\begin{array}{l}\text { Oil is supplied in the bearing for } \\
\text { every } 15 \text { seconds }\end{array}$ \\
\hline 4 & Fault 1 & $\begin{array}{l}\text { Grooving on the inner race of the } \\
\text { bearing is done using lathe ma- } \\
\text { chine }\end{array}$ \\
\hline 5 & Fault 2 & $\begin{array}{l}\text { A hole is made through the bear- } \\
\text { ing using drilling machine }\end{array}$ \\
\hline 6 & Fault 3 & $\begin{array}{l}\text { Indentations were made on the } \\
\text { inner race of the bearing using } \\
\text { grinding machine }\end{array}$ \\
\hline 7 & Full loose & $\begin{array}{l}\text { The screws holding the bearing } \\
\text { were almost completely loosened }\end{array}$ \\
\hline
\end{tabular}

Thus, the various types of faults that are induced on the bearing surface and the corresponding signals for each fault are recorded and analyzed through MATLAB software to check the severity of the damage.

\subsection{Signal processing}

Figure 3e shows the vibration measurement system developed for this research. It consists of an accelerometer, amplifier and personal computer. The vibration signal from the accelerometer is amplified and then the input signal is transferred to the computer. The amplified vibration waveforms are to be continuously transferred to the computer hard disk at the sampling rate of $12 \mathrm{KHz}$, for one complete revolution per minute. Fi- 
nally, the stored vibration signal can be processed using advanced signal processing methods to locate the faults in the bearing.

\section{Results and discussion}

A typical time domain signal obtained from the journal bearing test rig without any defect, using accelerometer is given in Fig. 3e. This is further processed using various signal processing techniques based on FFT and the principle of Daubechies Wavelet enveloped Power Spectrum Density (PSD) which is explained in the previous Sec. 2.

The PSD plot shows the frequency in $\mathrm{Hz}$ along the $x$-axis and the amplitude $g$ in $\mathrm{m} / \mathrm{s}^{2}$ along the $y$-axis. Figures 4 and 5 show the time domain signal and FFT signal of the bearing without faults. Figure 6 shows the Daubeuchies wavelet (DB) of bearing without faults and Fig. 7 shows filtered signal of the bearing without faults. From this Power Spectrum Density (PSD) plot, it can be observed that before wavelet decomposition the bearing frequency is $59.18 \mathrm{~Hz}$ for the shaft speed of $1240 \mathrm{rpm}$ and after the Daubechies Wavelet decomposition, the PSD plot shows a frequency of $20.65 \mathrm{~Hz}$.

The raw signal is fed into a system which performs wavelet transforms for DB02 wavelet (XU et al., 2009). This is done to obtain mechanical vibration range of frequencies and hence filter out the undesired garbage data. This helps increase accuracy of the system to a great extent (TiAnrui et al., 2013). The wavelet decomposition gives us a choice to pick

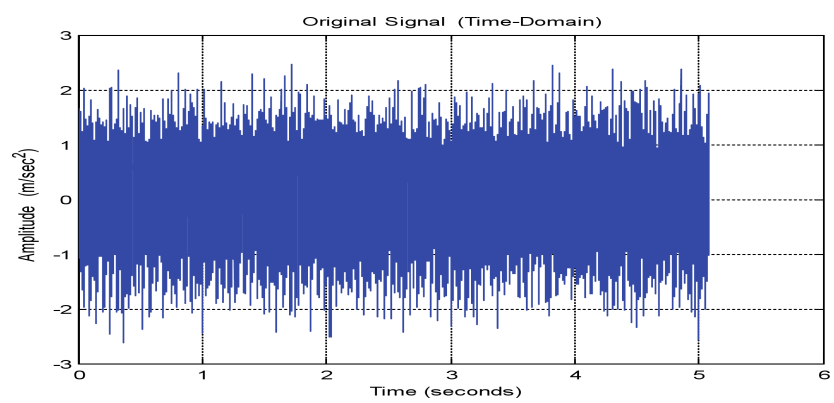

Fig. 4. Time domain signal of the bearing without faults.

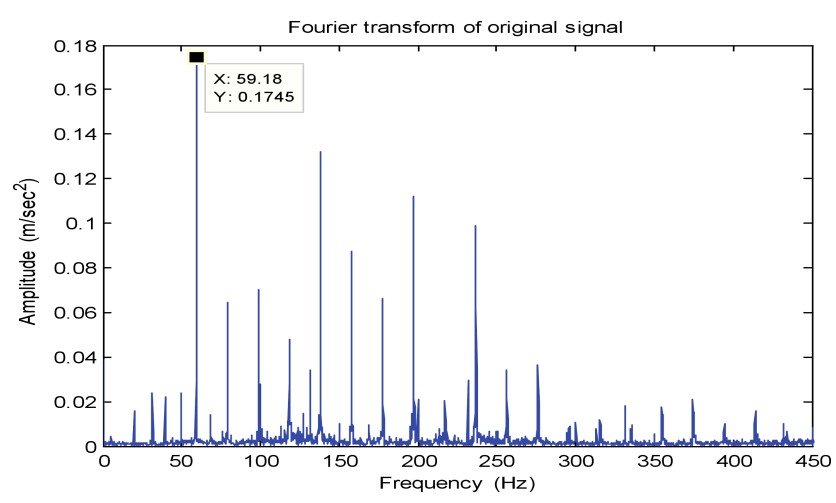

Fig. 5. FFT signal of the bearing without faults.

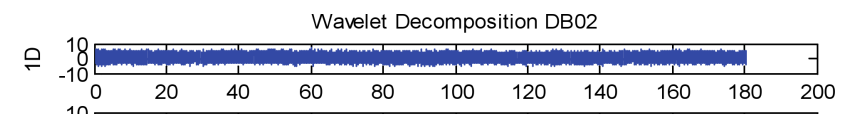

ลิ
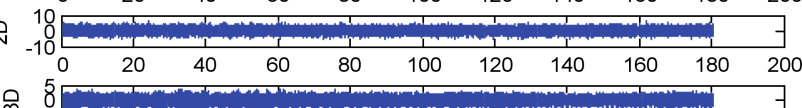

\begin{tabular}{lllllllllll}
2 & 0 \\
\hline & 20 & 40 & 60 & 80 & 100 & 120 & 140 & 160 & 180 & 200
\end{tabular}

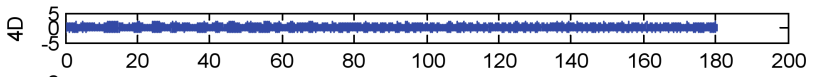

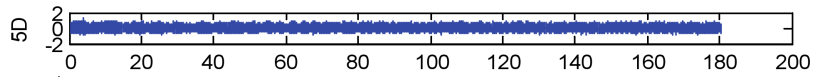

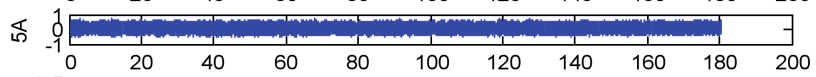

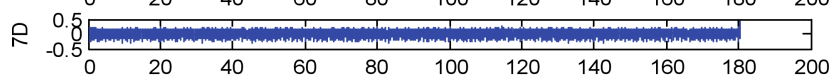

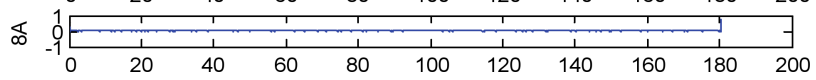

Fig. 6. Wavelet decomposition of the bearing without faults.

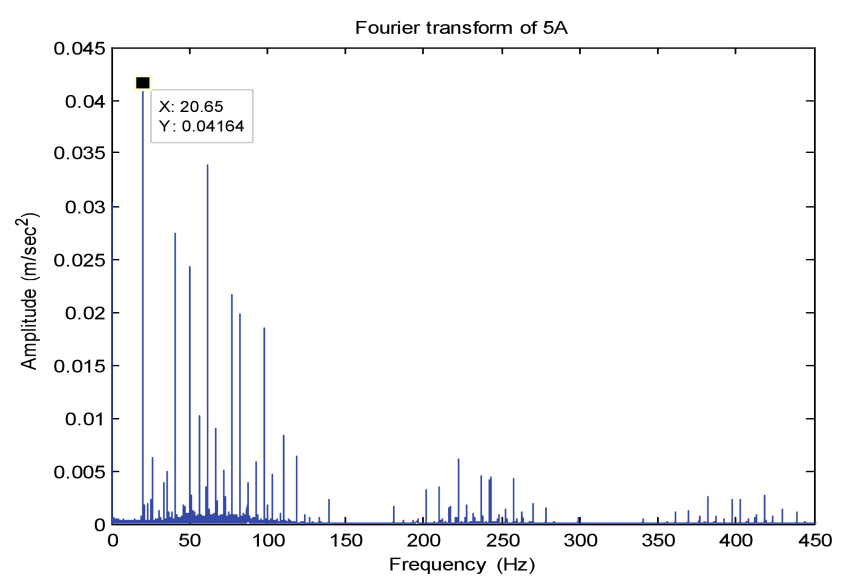

Fig. 7. Filtered signal of bearing without faults.

any of its decomposed frequencies as shown below in Fig. 6. As seen in the diagram we are given a choice of 8 classes of frequencies. Here D stands for 'detailed' while A stands for 'approximate'. The number refers to the degree of decomposition (TSE, 2004). The 5A decomposition, i.e 5th approximate decomposition is taken into the next step in the process. Reasons for the same have been described in further sections. The data (currently in time domain) is then operated upon by the fast Fourier transform to obtain the frequency domain. An example is shown in Fig. 5. Then the peaks are recorded along with the sub harmonics which are the next closest peaks to the main peak with the highest amplitude. Each of these readings is obtained for constant loads. Then a suitable format is decided and the readings are fed into a neural network with set training, validation and test samples. The accuracy of the neural network determines the existence of the pattern and also a classification of the particular fault.

Next, the peak frequency obtained was observed as a mismatch with the rps (rotations per second) value. Hence we had to code the wavelet transform in MAT- 
LAB to filter out the unwanted vibration and keep the mechanical frequencies which are obtained in the different patterns desired. It was then validated with the comparison as shown in Figs. 6 and 7.

From the results it was found that the after filtering the vibration data, the additional noise generated due to electrical interference during running conditions is reduced. There is a clear indication that there is no fault. But, through the Fast Fourier Transform (FFT) signal plot it is very difficult to locate the exact fault.

Figure 7 depicts the important features of frequency content including the shaft frequency of the vibration signal at $1240 \mathrm{rpm}(20.65 \mathrm{~Hz})$. There is no indication of a fault in the frequency spectrum.

In the journal bearings, mechanical looseness can develop at operating speeds and at high temperatures. First, half looseness is created on the bearing casing and the signals are recorded through the experimental setup. Figures 8 and 9 show time domain signal of the bearing at half looseness and FFT signal of the bearing at half looseness. Figure 10 shows the DB02 decomposition of the bearing and Fig. 11 shows the filtered signal of the bearing at half looseness. From this Power Spectrum Density (PSD) plot, it is observed that the bearing frequency for half mechanical looseness is $60.36 \mathrm{~Hz}$ with the peak amplitude of 0.09049 . This increased frequency is due to the combination of sub harmonics and interharmonics for mechanical looseness of the bearing.

By comparing the time domain signals of Figs. 8, $12,16,20,24$, and 28 we can highlight a few waveforms showing the varying degrees of amplitude of the

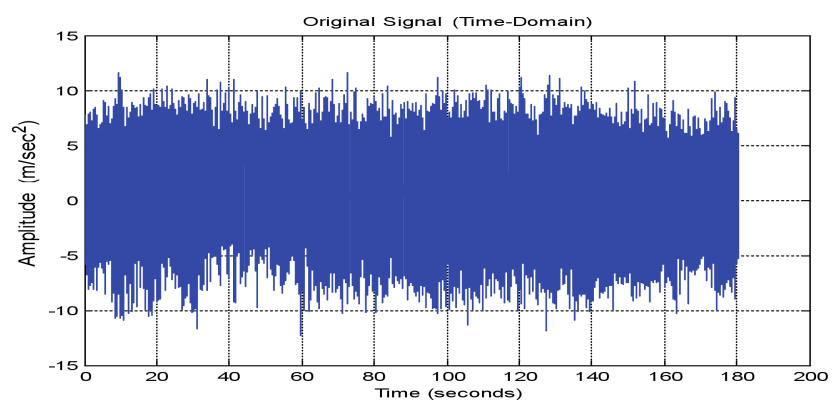

Fig. 8. Time domain signal of the bearing at half looseness.

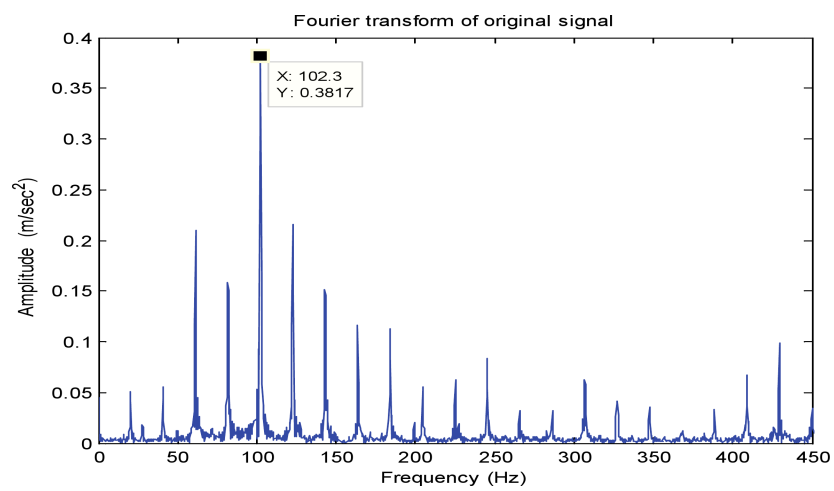

Fig. 9. FFT signal of the bearing at half looseness.

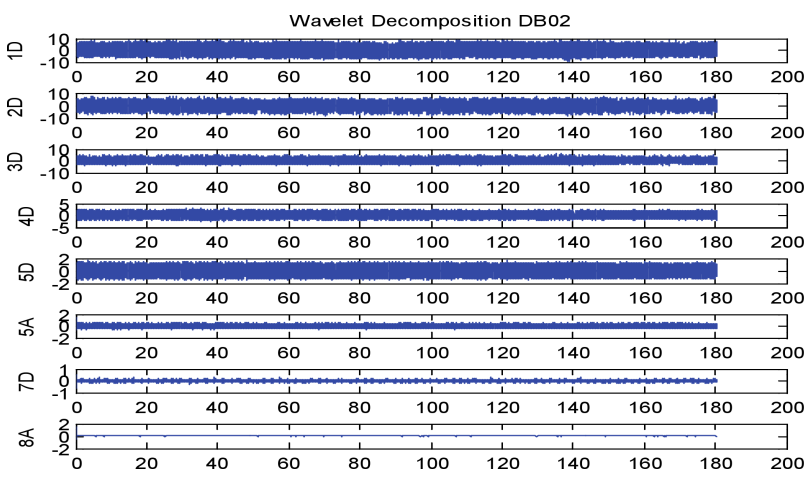

Fig. 10. Wavelet decomposition of the bearing at half looseness.

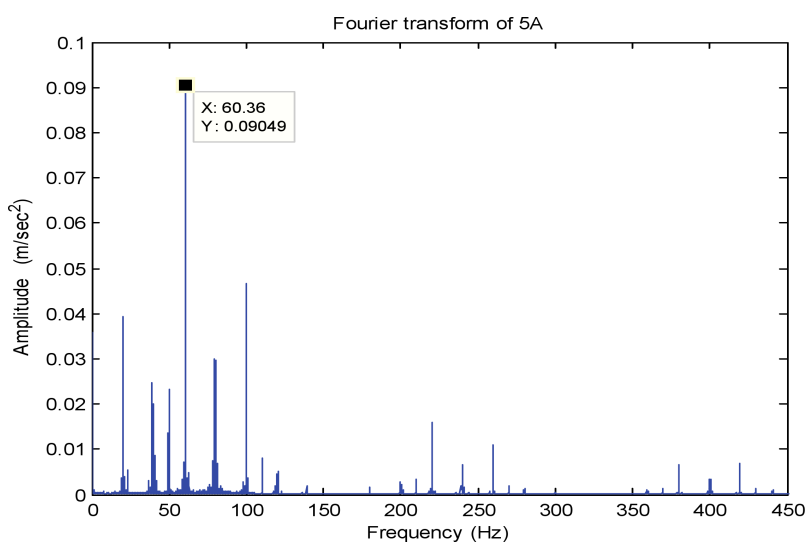

Fig. 11. Filtered signal of the bearing at half looseness.

modulated waveform which proved to be a sign of the intensity of the fault. These observations validate the results of indication of a fault contact within the journal. Thus, a vibration signature modulated at the rotational speed of the unit is illustrative of a continuous source of fault. This fault implies a sustained contact between the shaft and the bearing generating vibration levels above the operational background levels.

By comparing the power spectrum shown in Figs. 7 and 11 it can be found that the amplitudes of the friction frequency and its harmonics increase with the increase in misalignment conditions. This accelerates the shaft causing it to rebound from the surface at $20.65 \mathrm{~Hz}$ vibrations in frequency spectrums. In the journal bearings, full looseness may only develop at high speeds and temperatures. Then the full looseness is provided on the bearing casing and the signals are recorded through the experimental setup. Figures 12 and 13 show time domain signal of the bearing at full looseness and FFT signal of the bearing at full looseness. Figure 14 shows the wavelet decomposition (DB02) of the bearing at full looseness and Fig. 15 shows the filtered signal of the bearing at full looseness.

From this Power Spectrum Density (PSD) plot, before wavelet decomposition (DB02) of the bearing, it was observed that the bearing frequency for full looseness was $118.4 \mathrm{~Hz}$ with the peak amplitude of 0.4629. After DB02 decomposition, the PSD plot 


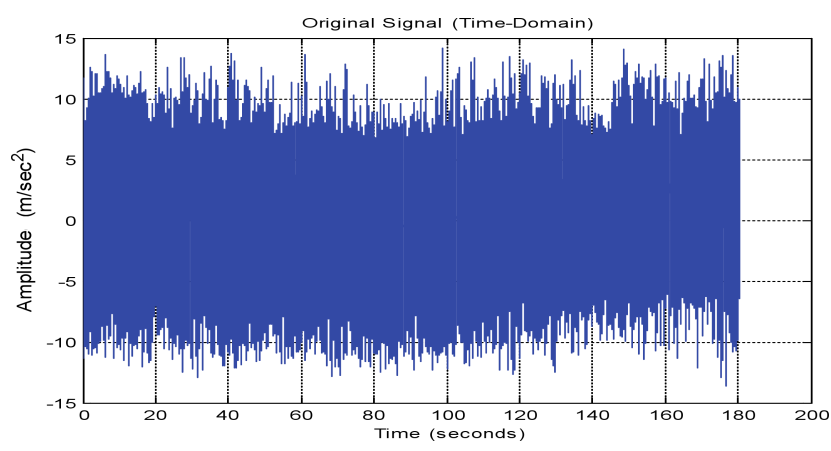

Fig. 12. Time domain signal of the bearing at full looseness.

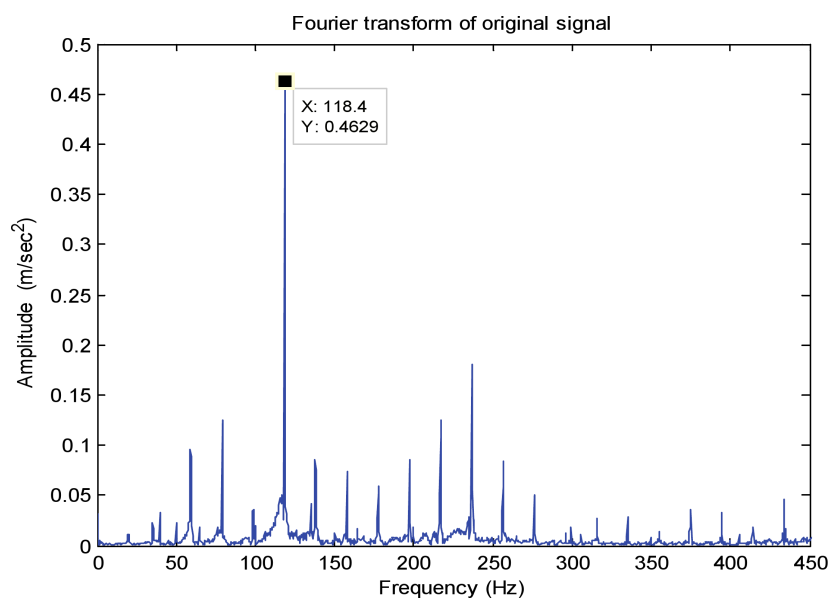

Fig. 13. FFT signal of the bearing at full looseness.

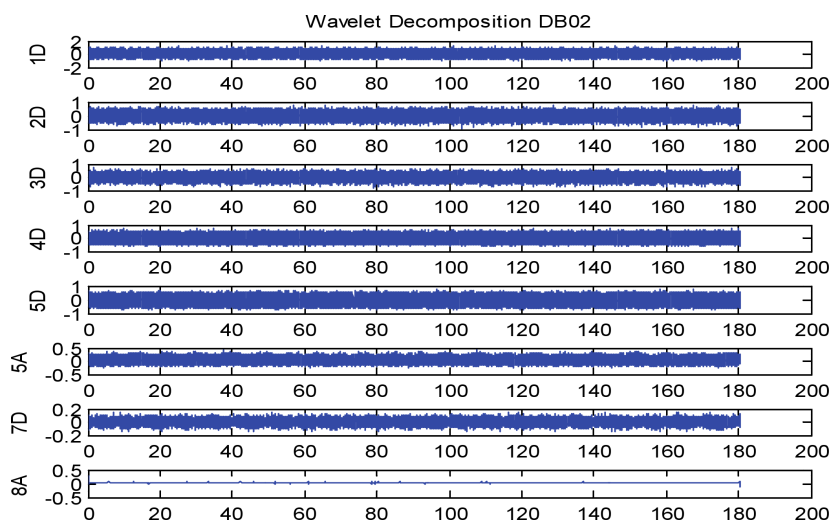

Fig. 14. Wavelet decomposition of the bearing at full looseness.

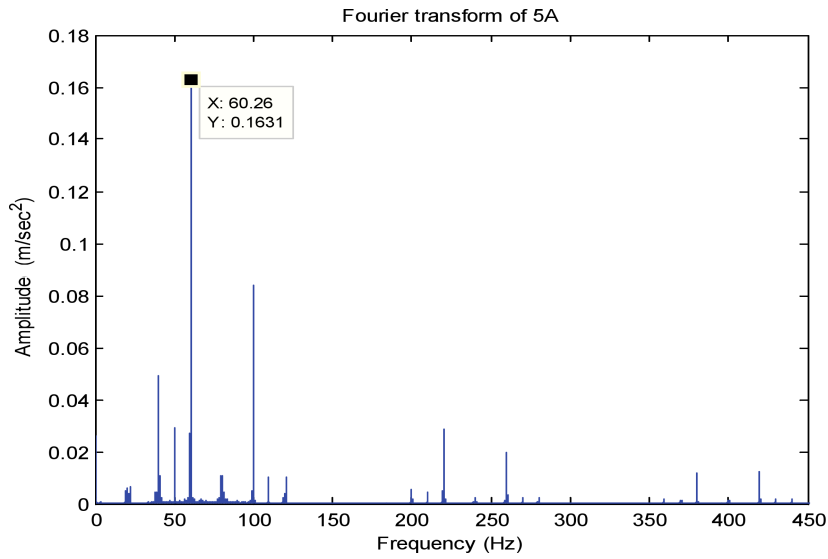

Fig. 15. Filtered signal of the bearing at full looseness. shows the frequency of $60.26 \mathrm{~Hz}$ with the peak amplitude of 0.1631. Thus, after DB02 decomposition, unwanted vibration generated from the experimental setup is filtered out and hence considerable peak amplitude is reduced due to the electrical interference of motor.

When compared to the half looseness, an increase in peak amplitude is observed at the full looseness and hence it generates more vibration and noise. From the result it can be seen that there is an increase in the severity of the looseness.

Generally, in the journal bearings, half lubrication behaviour occurred, due to side leakages of oil between the shaft and the bearing and further it can deteriorate the inner surface of the bearing. This will decrease eccentricity, increase the oil temperature and reduce viscosity. In the journal bearings, oil instability may only develop at side leakages and poor mechanical maintenance. Using the experimental set up, the half lubrication is poured through the oil hole provided on the bearing casing and the signals are recorded.

The coefficient of friction gradually starts to increase between the shaft and the bearing due to improper lubrication. Figures 16 and 17 show time domain signal of the bearing at half lubrication and FFT signal of the bearing at half lubrication. Figure 18 shows the DB02 decomposition of the bearing at half

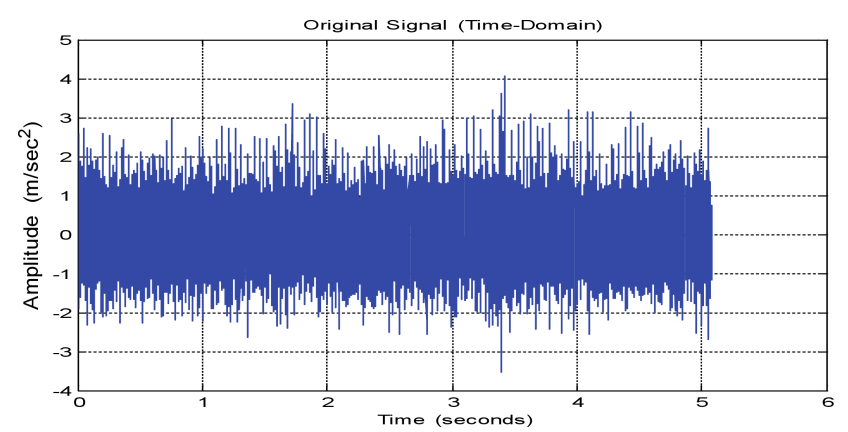

Fig. 16. Time domain signal of the bearing at half oil.

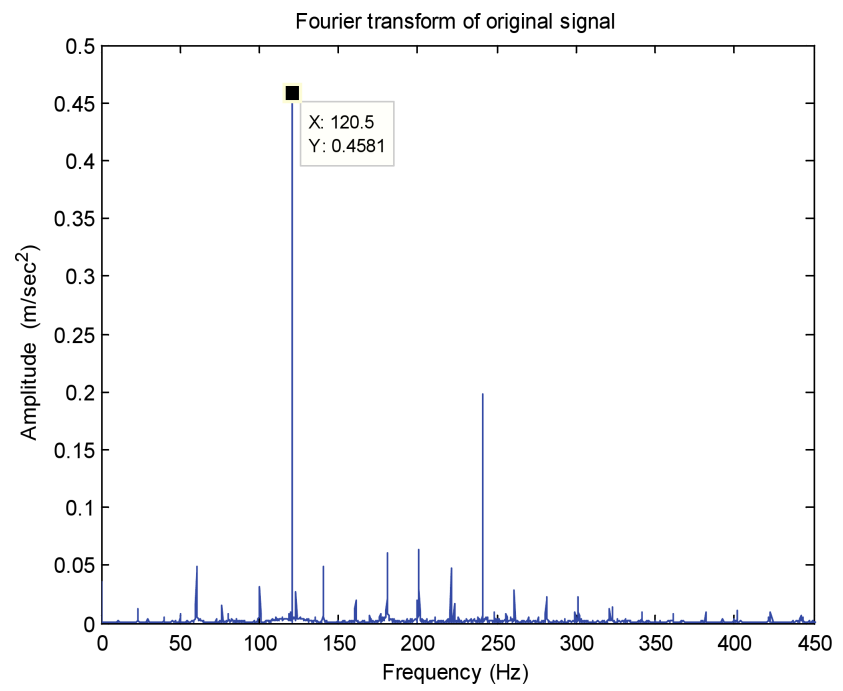

Fig. 17. FFT signal of the bearing at half oil. 
lubrication and Fig. 19 shows filtered signal of the bearing at half lubrication. From this Power Spectrum Density (PSD) plot, it is observed that the bearing frequency at half lubrication is $102 \mathrm{~Hz}$.

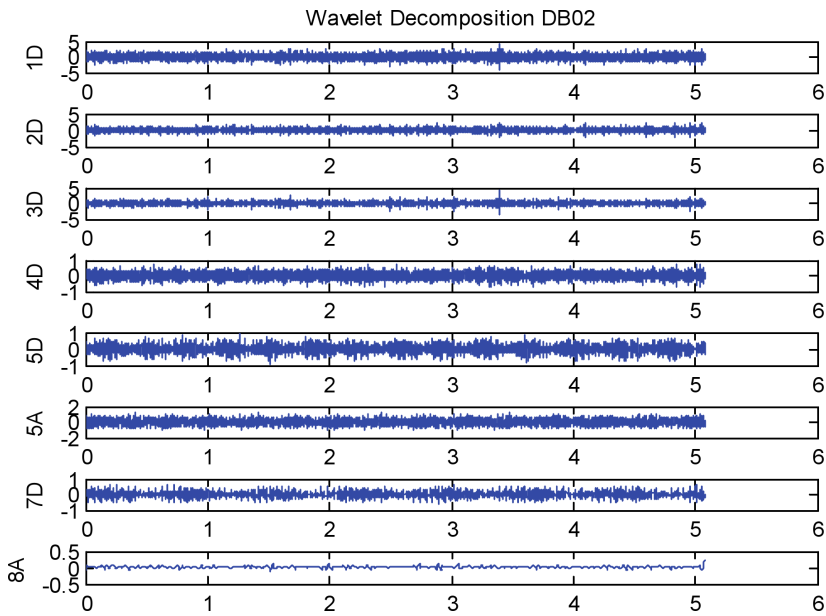

Fig. 18. Wavelet decomposition of the bearing at half oil.

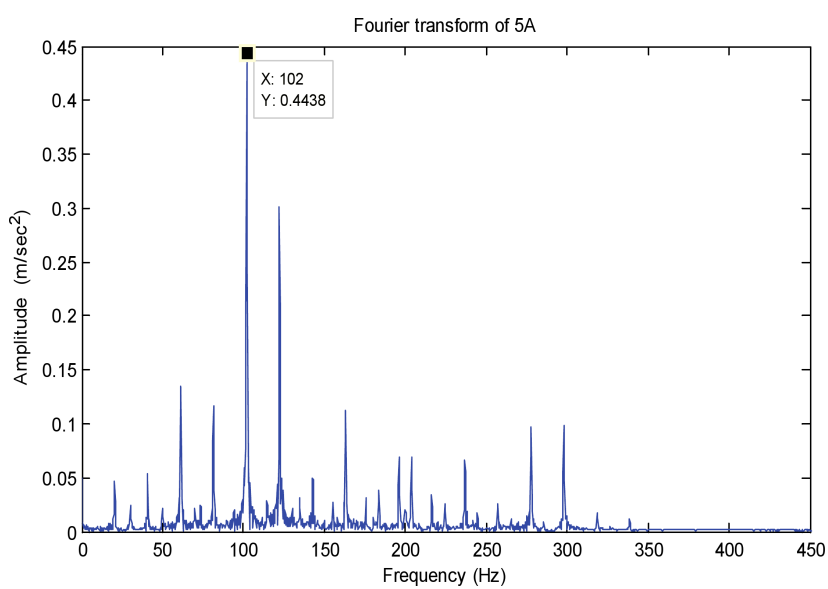

Fig. 19. Filtered signal of the bearing at half oil.

First the eccentric bore fault (fault 1) was created as shown in Fig. 4 and the vibrations data were recorded at a shaft speed of $1240 \mathrm{rpm}$. Figures 20 and 21 show time domain signal of the bearing at fault 1 and FFT signal of the bearing at fault 1. Figure 22 shows the wavelet decomposition of the bearing at fault 1 and Fig. 23 shows the filtered signal of the bearing at fault 1. From this Power Spectrum Density (PSD) plot, it was observed that the bearing frequency for fault 1 was $119.9 \mathrm{~Hz}$ which is greater than the frequency of healthy bearing which is $20.65 \mathrm{~Hz}$. This shows that a fault was induced on the bearing.

The eccentric bore fault (fault 1) and the fault with a hole (fault 2) develops a strong harmonics of the shaft rotational speed in the vibration analysis. In fault simulation, once the shaft and bearing are brought in contact then the stiffness of the test rig increases. This fault behaviour tends to produce strong impulses between the journal and bearing which is used to excite

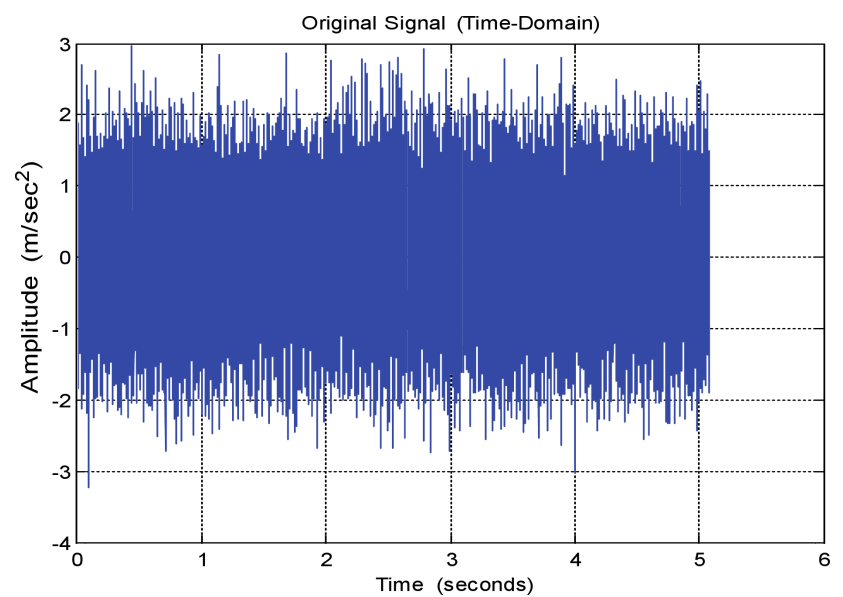

Fig. 20. Time domain signal of the bearing at fault 1 .

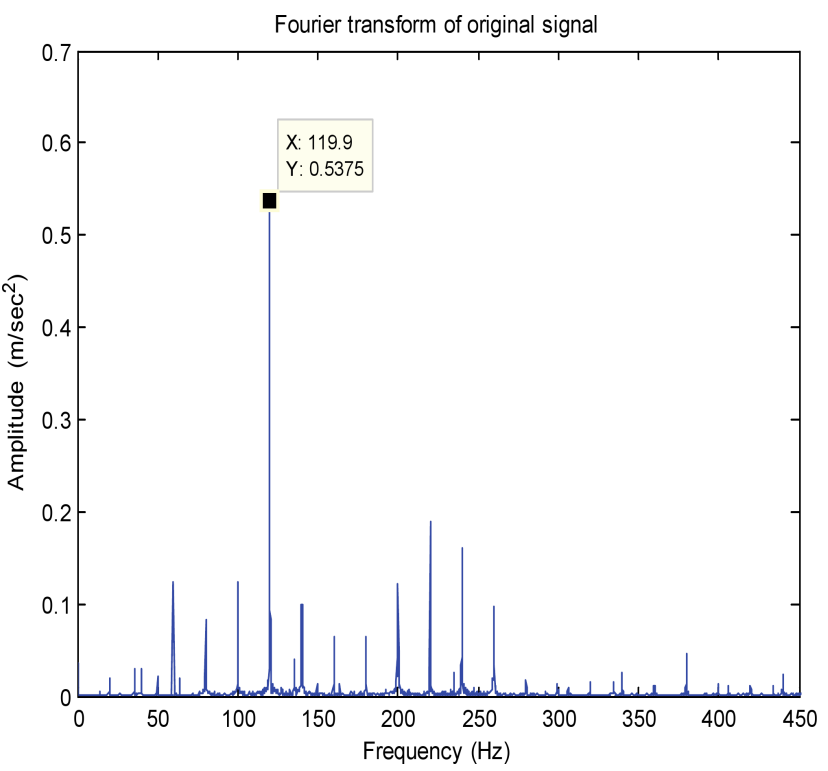

Fig. 21. FFT signal of the bearing at fault 1 .

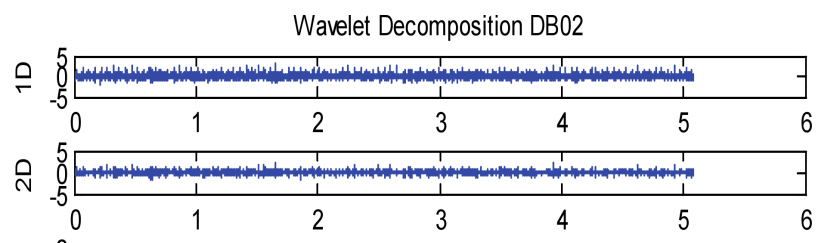

仓 $\int_{-2}^{2}$

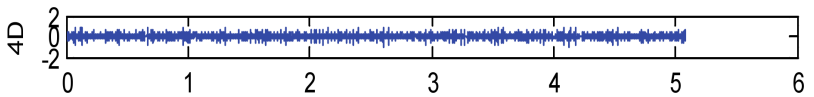

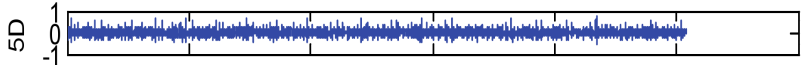
$\begin{array}{rrrrrrr}-1 & 1 & 2 & 3 & 4 & 5 & 6\end{array}$

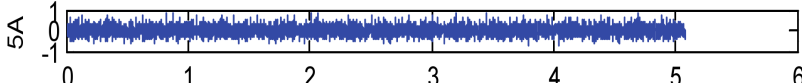

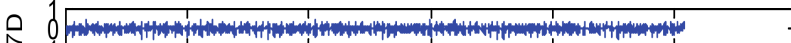
$\begin{array}{rrrrrrr}-1 & 1 & 2 & 3 & 4 & 5 & 6\end{array}$

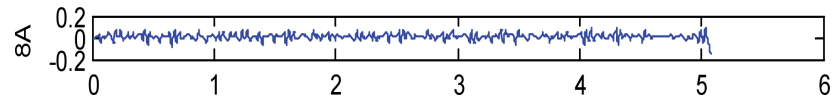

Fig. 22. Wavelet decomposition of the bearing at fault 1 . 


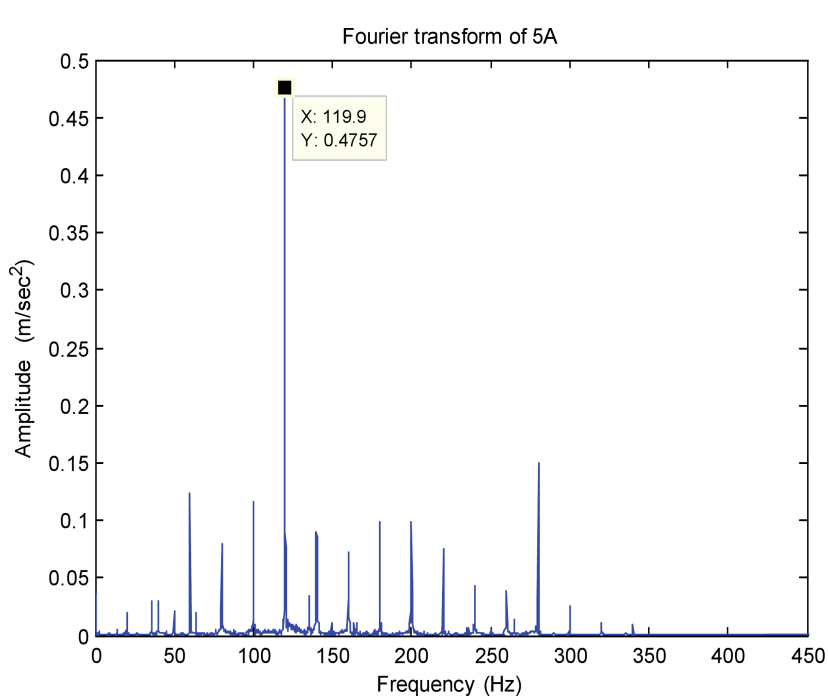

Fig. 23. Filtered signal of the bearing at fault 1 .

the system resonance and in addition creates strong harmonics.

From Figs. 23 and 27, it was found that the dominant frequency of the vibration signals for fault1 and fault 2 conditions were registered to be approximately $119.9 \mathrm{~Hz}$ and $121.5 \mathrm{~Hz}$ respectively. The Nonsynchronous peaks on the frequency spectrum are an indication of bearing fault. The increase in amplitude of these frequencies indicates the change of fault. As the fault increases, the frequency of these peaks develops harmonics, indicative of impacting. Next the eccentric bore fault with a crack of $3 \mathrm{~mm}$ (fault 2) shown in Fig. 6 was created and the readings were recorded at a shaft speed of $1240 \mathrm{rpm}$. Figures 24 and 25 show time domain signal of the bearing at fault 2 and FFT signal of the bearing at fault 2. Figure 26 shows the DB02 wavelet decomposition of the bearing before applying the filter at fault 2 and Fig. 27 shows the filtered signal of the bearing at fault 2 .

From the results of PSD plot, it was observed that the bearing frequency for fault 2 was $121.5 \mathrm{~Hz}$ which is greater than the healthy bearing of $20.65 \mathrm{~Hz}$. The

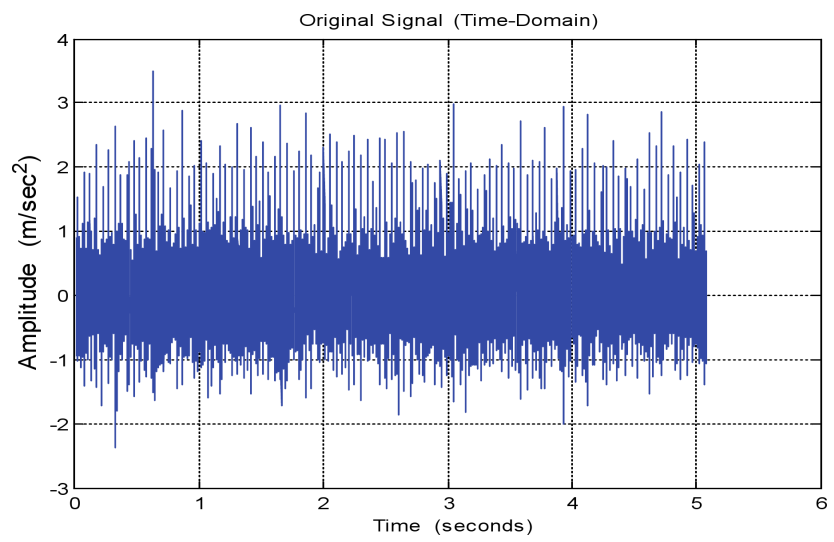

Fig. 24. Time domain signal of the bearing at fault 2 .

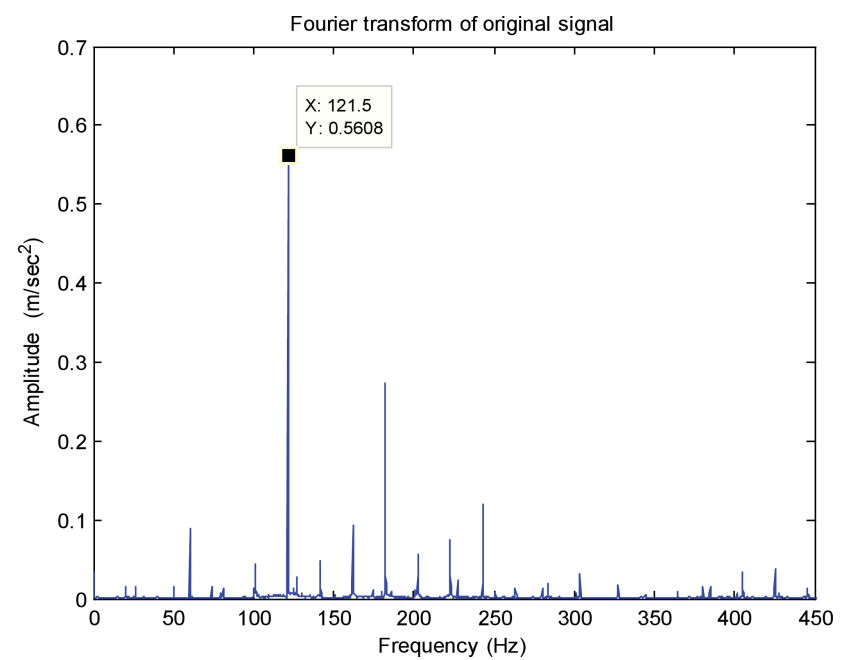

Fig. 25. FFT signal of the bearing at fault 2 .

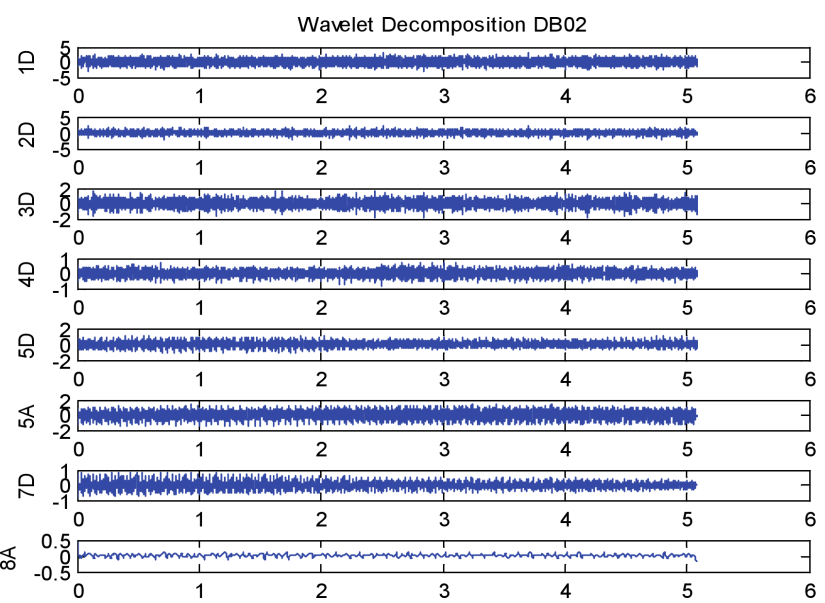

Fig. 26. Wavelet decomposition of the bearing at fault 2 .

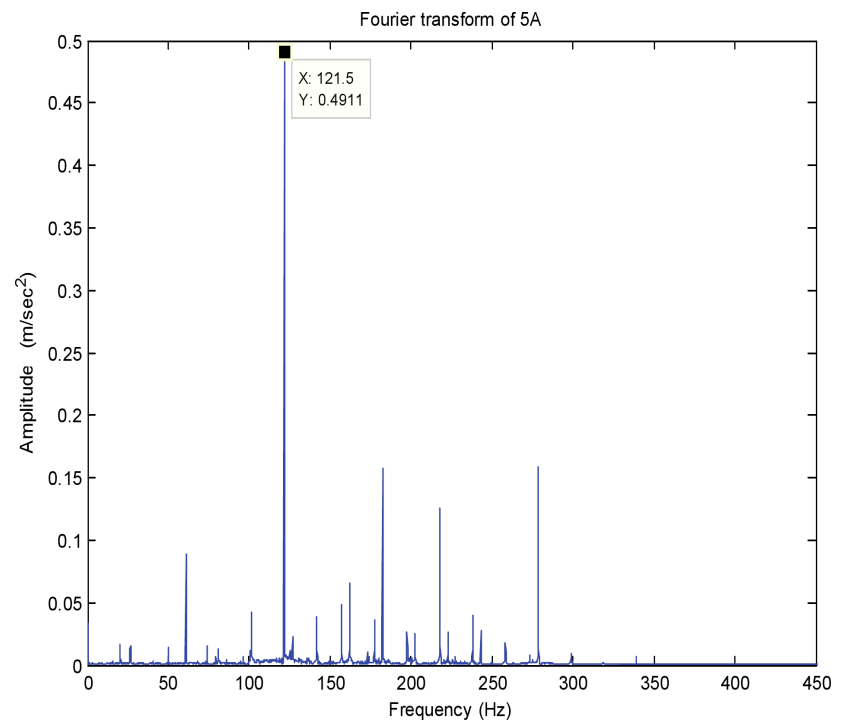

Fig. 27. Filtered signal of the bearing at fault 2 .

PSD plots show more amplitude and an increase in frequency was observed. From the result it can be seen that there was increase in the severity of the faults when compared to the fault 1. 
Figure $3 \mathrm{~d}$ is the photograph depicting fault 3 along with fault 1 and fault 2 . Fault 3 was created by removing the material on the surface of the bearing using grinding machine. The vibration signals were recorded at a shaft speed of $1240 \mathrm{rpm}$. Figures 28 and 29 show time domain signal of the bearing at fault 3 and FFT signal of the bearing at fault 3 . Figure 30 shows the wavelet decomposition (DB02) of the bearing at fault 3 and Fig. 31 shows the filtered signal of the bearing at fault 3 .

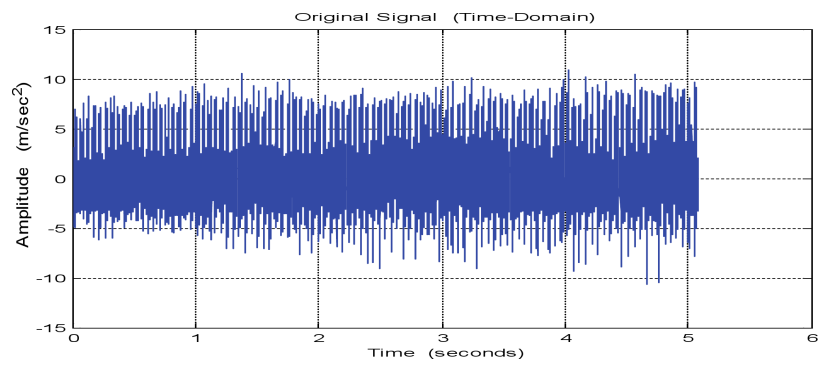

Fig. 28. Time domain signal of the bearing at fault 3 .

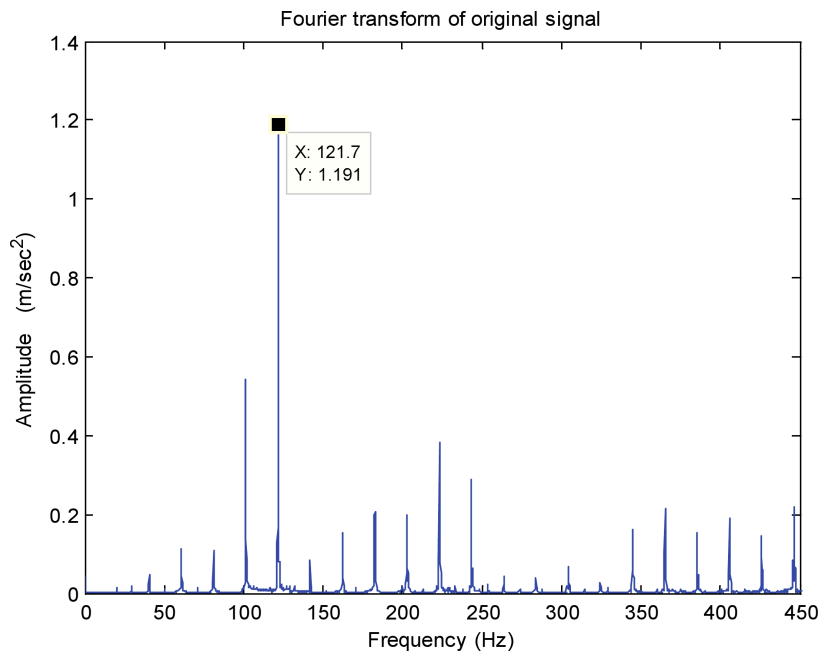

Fig. 29. FFT signal of the bearing at fault 3 .
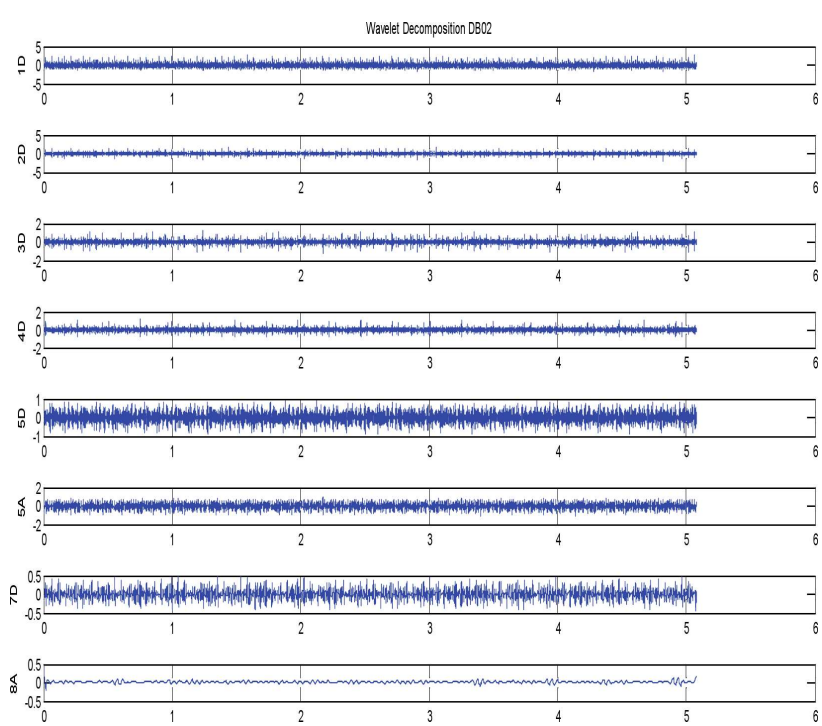

Fig. 30. Wavelet decomposition of the bearing at fault 3 .

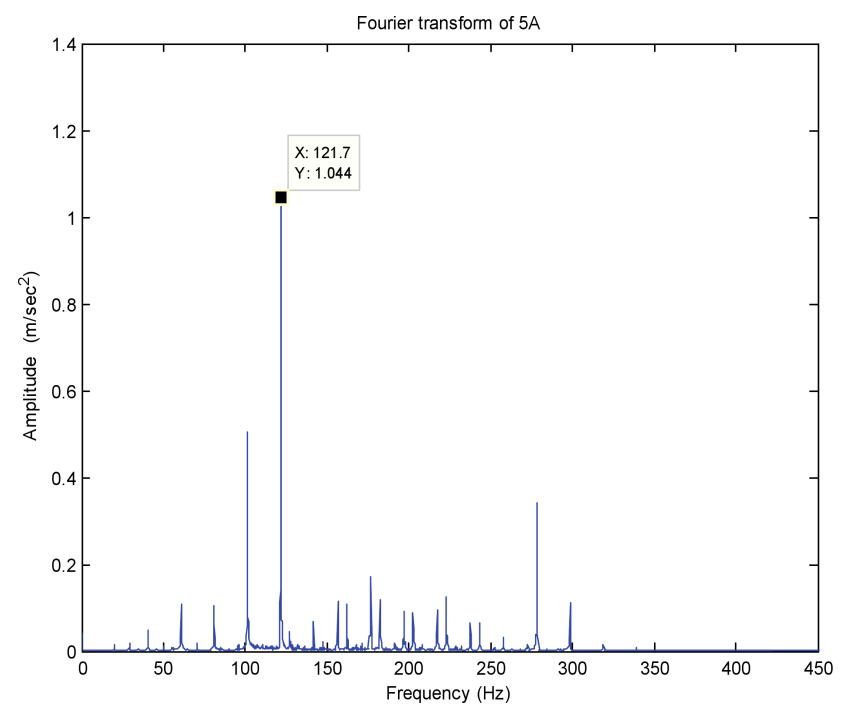

Fig. 31. Filtered signal of the bearing at fault 3.

By enhancing the fault (material removal fault 3) on the bearing, the frequency is increased, to the high frequency side of the spectrum. This shows that the various rotating components involved in the experimental set up hardens as a result of the stiffness produced by the contact of the shaft with bearing. From Fig. 31 it is observed that the frequency increases as a result of the fault impacts. The enveloping analysis demonstrates that the dominant frequency of the vibration signals for fault 3 were registered to be $121.7 \mathrm{~Hz}$ with the amplitude of vibration $1.044 \mathrm{~m} / \mathrm{s}^{2}$. Table 3 shows the results of FFT and Table 4 shows the results of filtered signal.

Table 3. Results of FFT.

\begin{tabular}{|c|c|c|c|}
\hline S. No. & $\begin{array}{c}\text { Conditions } \\
\text { of the journal bearing }\end{array}$ & $\begin{array}{c}\text { Frequency } \\
{[\mathrm{Hz}]}\end{array}$ & $\begin{array}{c}\text { Amplitude } \\
{\left[\mathrm{m} / \mathrm{s}^{2}\right]}\end{array}$ \\
\hline 1 & Healthy bearing (no fault) & 59.18 & 0.1745 \\
\hline 2 & Half looseness & 102.3 & 0.3817 \\
\hline 3 & Full looseness & 118.4 & 0.4629 \\
\hline 4 & Half oil & 120.5 & 0.4581 \\
\hline 5 & Fault 1 & 119.9 & 0.5375 \\
\hline 6 & Fault 2 & 121.5 & 0.5608 \\
\hline 7 & Fault 3 & 121.7 & 1.191 \\
\hline
\end{tabular}

The identification and the monitoring of the bearing fault using the spectral analysis are difficult, due to the non-stationary. To extract the fault information, the DB-02 wavelet is applied to the vibration signals. The result shown in Table 1 shows that as the fault increases the frequency and amplitude are also increased. This shows that the strong harmonics is developed when the fault is generated on the bearing. From the above table, the healthy bearing has the frequency of $20.65 \mathrm{~Hz}$ with the amplitude of $0.04164 \mathrm{~m} / \mathrm{s}^{2}$ and for fault1, fault 2 and fault 3 the frequencies are of 
Table 4. Results of filtered signal.

\begin{tabular}{|c|c|c|c|}
\hline S. No. & $\begin{array}{c}\text { Conditions } \\
\text { of the journal bearing }\end{array}$ & $\begin{array}{c}\text { Frequency } \\
{[\mathrm{Hz}]}\end{array}$ & $\begin{array}{c}\text { Amplitude } \\
{\left[\mathrm{m} / \mathrm{s}^{2}\right]}\end{array}$ \\
\hline 1 & Healthy bearing (no fault) & 20.65 & 0.04164 \\
\hline 2 & Half looseness & 60.36 & 0.09049 \\
\hline 3 & Full looseness & 60.26 & 0.1631 \\
\hline 4 & Half oil & 102 & 0.4438 \\
\hline 5 & Fault 1 & 119 & 0.4757 \\
\hline 6 & Fault 2 & 121.5 & 0.4911 \\
\hline 7 & Fault 3 & 121.7 & 1.044 \\
\hline
\end{tabular}

$119,121.5$ and $121.7 \mathrm{~Hz}$ with the increase in amplitude of $0.4757,0.4911$ and $1.044 \mathrm{~m} / \mathrm{s}^{2}$, respectively. When comparing the results of FFT in Table 3, the filtered signal shows the reduced peak amplitudes in Table 4. This clearly indicates the bearing fault. Also, from the result it can be seen that there is an increase in the severity of the faults. When compared to fault 2 , more peaks and an increase in frequency were observed.

\section{Artificial Neural Networks}

A feed forward Multi Layer Perceptron (MLP) neural network which consists of three layers has been developed. The input layer of six source nodes represents the normalized features extracted from the time domain. A hidden layer with five computation nodes has been used. The number of hidden nodes is optimized by minimization of Mean Square Error (MSE) between the actual network outputs and the corresponding target values. The output layer with two nodes indicates the different working conditions (healthy and faulty) of the bearings which require to be identified by the neural network. The two digit output target nodes that need to be mapped through the ANN which is able to classify the working condition of healthy bearing as $(0,1)$ and faulty as $(1,0)$ has been developed. Figure 32 shows the architecture of ANN of the proposed diagnostic system. In ANN, 70\% of data subset is used for training, $15 \%$ of data for validation and $15 \%$ of data for testing.

The training of Multi Layer Perceptron network is achieved by modifying the connection weights and biases iteratively to optimize the performance crite-

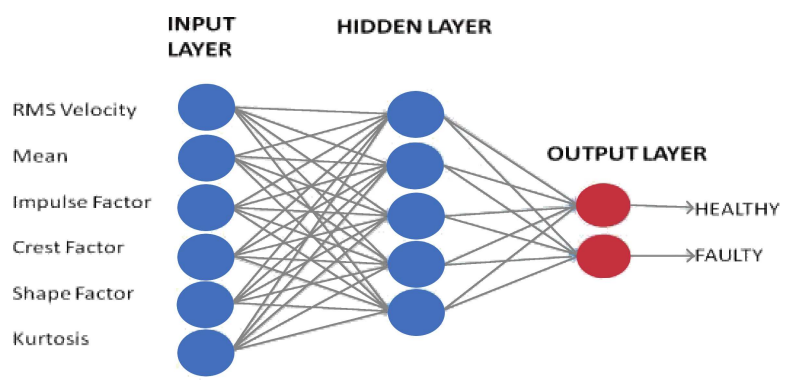

Fig. 32. Architecture of ANN. rion. The performance criterion is the minimization of the Mean Square Error (MSE) between the actual network output and the corresponding target values in the training set. The training algorithms for Multi Layer Perceptron are based on Back Propagation (BP). The Back Propagation adapts a gradient descent approach by adjusting the ANN connection weights.

The following techniques were applied to enhance the network performance and among them, the network which produces the lowest validation error during training was selected as the optimum network. For each network with a specific size of layer, the network weights and biases were reset three times by initiate and train procedure to obtain new values. Then, with respect to the minimum number of required frequencies for the minimum classification error, the best iteration of the loop or the optimum network was selected and through this the most suitable frequencies among all of the initial frequencies were obtained.

The Mean Square Error is achieved through Back Propagation in the network and is used to adjust the connection weights between the layers, thus improving the network classification performance. This process is repeated until the overall Mean Square Error value drops below some predetermined threshold shown in Fig. 33. After achieving the training process, the ANN weights are fixed and the system is deployed to solve the journal bearing condition identification problem using unseen vibration data.

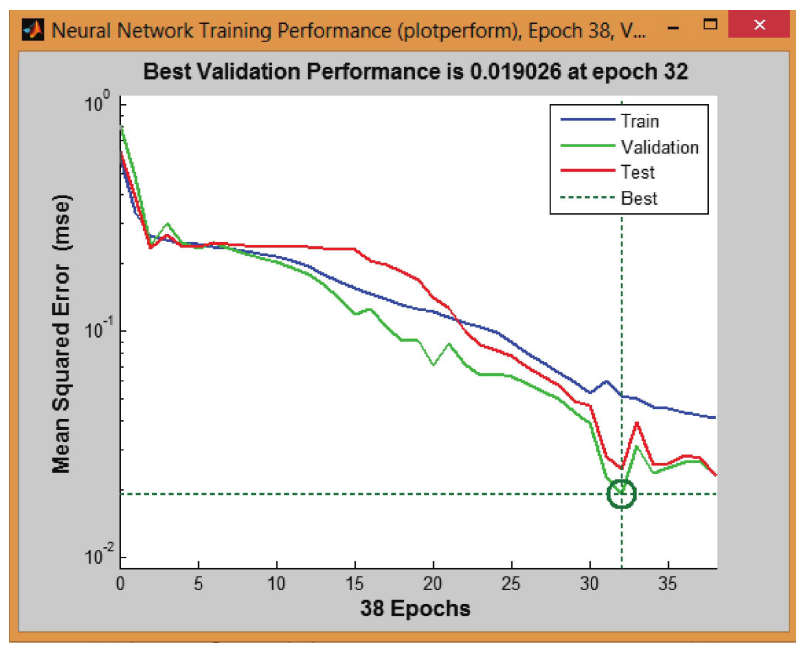

Fig. 33. Performance graph for fault classification.

The Artificial Neural Network (ANN) was created, trained and tested using MATLAB Neural Network Toolbox with Levenberg-Marquarat Back Propagation (LMBP) training Algorithm. In this work, statistical features like mean, impulse factor, shape factor, crest factor, kurtosis and Root Mean Square (RMS) of vibration signals were used as input elements to the ANN and MSE of $10 \mathrm{E}-1$, with a minimum gradient of $10 \mathrm{E}-1$ and maximum iteration(epochs) of 1000. The train- 
ing process would have stopped if any of these conditions had been met. The initial weights and biases of the network were randomly generated by the program. The ANN needs only 38 epochs to reach the required goal of $10^{-1} \mathrm{MSE}$. The MSE for the testing process is shown in Fig. 33. This is the average square difference (error) between normalized output and the target. When the MSE is zero, it means that there is no error in the training process, but if MSE is greater than 0.5 it means that the error is higher. Therefore, for a good training result, MSE should be within the range. Figure 33 show the MSE (Performance graph) of the ANN trained for fault detection. However, the MSE of the three stages are approximately zero. This means that, the ANN selected for the three stages of diagnosis were correct and was successfully used for the three tasks.

The Neural Network test process for unseen vibration data of the trained ANN combined with the ideal output target values is presented. This indicates the high success rate of $85.7 \%$ classification for journal bearing fault detection and classification in Fig. 35. From the classified values shown in Table 5, it is observed that there is no error for healthy and faulty bearings.
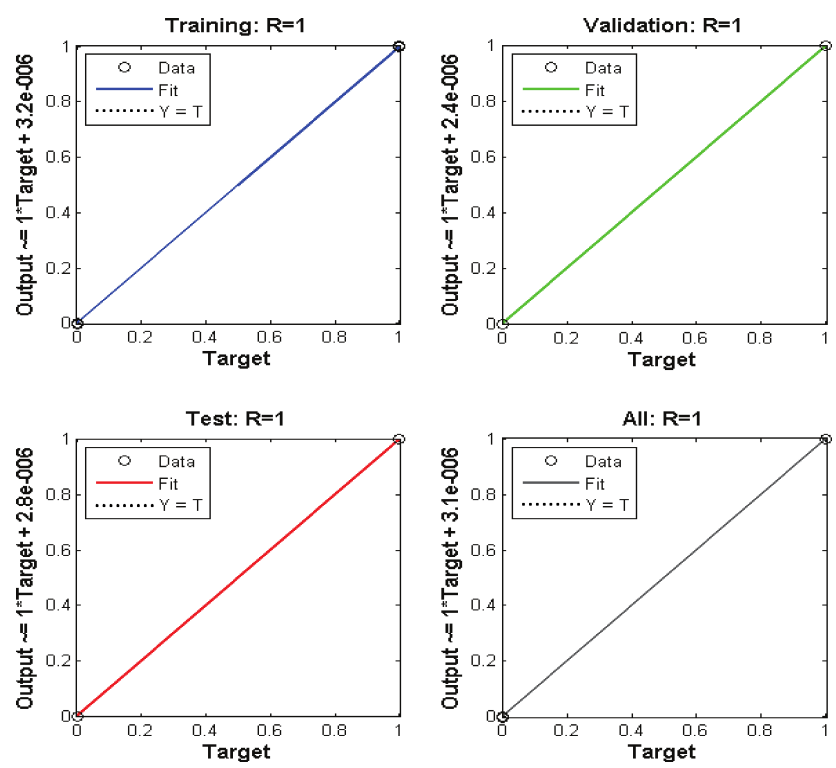

Fig. 34. Regression graph for fault detection.

Table 5. Classified values $\mathrm{R}^{2}$.

\begin{tabular}{|c|c|c|c|c|c|}
\hline 0.002 & 0.0011 & 0.0013 & 0.0016 & 1 & 1 \\
\hline 1 & 1 & 1 & 1 & 0.031 & 0.021 \\
\hline
\end{tabular}

The regression graph was used in analyzing the training and the output of the network. The regression is a measure of the correlation between the unnormalized output and the targets. Thus, if Regression $\mathrm{R}$ is 1 , it means that there is a close relationship between the output and the target (i.e. their values tend to converge to the same value). At this point training can stop.

Figure 35 shows the confusion matrix. The confusion matrix shows that the $85.7 \%$ of faults on the bearing were classified. As a result, we can say that, the classification of faults on the bearing was successfully done using the selected ANN.

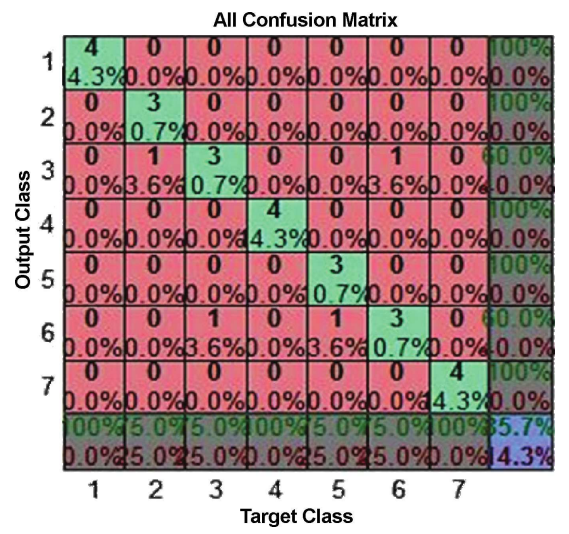

Fig. 35. Confusion matrix.

Thus, the graph tells us about the difference between the target and output of the selected network for the isolation of fault. If the difference is large, it means that, we will continue the training, but if it is small as seen in Fig. 33 the training will stop. This means that the network used for the isolation is correct.

\section{Conclusion}

From the experimental work, it can be seen that it is more advantageous to use DB02 wavelet technique in high rotational speed machines, rather than the traditional ones, because the results obtained through FFT were not capable of detecting the faults in the journal bearing test rig. Half lubrication was facilitated on the journal bearing. The enveloped Daubeuchies wavelet proved to be a valuable tool in conjunction with routine vibration data to provide a complete picture of the health of the rotating machinery. Thus, the condition monitoring proved to be effective in the early diagnosis of bearing faults. The results showed that various fault vibration signals and the fault detection rate of the proposed method is superior to FFT. Hence, this Daubeuchies wavelet technique would produce considerable savings by reducing unwanted external and internal noises of machinery. So, an industry can benefit immensely by condition monitoring. By using FFT, the maximum amplitude of vibration is $1.191 \mathrm{~m} / \mathrm{s}^{2}$ for fault 3, whereas maximum amplitude of vibration is $1.044 \mathrm{~m} / \mathrm{s}^{2}$ using DB-02 wavelet. The result shows that the amplitude of vibration is approximately $12 \%$ less in wavelet technique when compared to FFT technique. The Artificial Neural Network (ANN) was created, trained and tested using Neural Network Toolbox with Levenberg-Marquarat Back Propagation (LMBP) 
training Algorithm. The test process for unseen vibration data of the trained ANN combined with the ideal output target values indicated the high rate of success for automated bearing fault detection. Results, indicates the high success rate of $85.7 \%$ classification for journal bearing fault detection and classification.

\section{References}

1. Ali J.B., Fnaiech N., Saidi L., Chebel-MorelLO B., FNAiech F. (2015), Application of empirical mode decomposition and artificial neural network for automatic bearing fault diagnosis based on vibration signals, Applied Acoustics, 89, 2, 16-27.

2. Chen J., Li Z., Pan J., Chen G., Zi Y., Yuan J., Chen B., He Z. (2016), Wavelet transform based on inner product in fault diagnosis of rotating machinery: A review, Mechanical Systems and Signal Processing, 70-71, 1, 1-35.

3. DĄBrowski Z., Dziurdź J. (2016), Simultaneous analysis of noise and vibration of machines in vibroacoustic diagnostics, Archives of Acoustics, 41, 4, 783789 .

4. De Azevedo H., Araújo A.M., Bouchonneau N. (2016), A review of wind turbine bearing condition monitoring: State of the art and challenges, Renewable and Sustainable Energy Reviews, 56, 2, 368-379.

5. Dehm-Andone G., Mzyk R., Hausknecht F., Fisher G., Weigel R., Koelpin A. (2012), Filter design aspects in analog receiver front-ends for frequency scanning applications, International Symposium on Signals, Systems, and Electronics (ISSSE).

6. Feng K., Jiang Z., He W., Qin Q. (2011), Rolling element bearing fault detection based on optimal antisymmetric real Laplace wavelet, Measurement, 44, 9, $1582-1591$.

7. Guo P., INFIELD D. (2012), Wind turbine tower vibration modeling and monitoring by the nonlinear state estimation technique (NSET), Energies, 5, 12, 52795293.

8. HAKIM S.J.S., RAZAK H.A. (2014), Modal parameters based structural damage detection using artificial neural networks - a review, Smart Structures and Systems, 14, 2, 159-189, doi: 10.12989/sss.2014.14.2.159.

9. Hariharan V., Srinivasan P.S.S. (2012), New approach of classification of rolling element bearing fault using artificial neural network, Elixir Mechanical Engineering, 49, 1, 9964-9980.

10. Kankar P.K., Sharma S.C., Harsha S.P. (2011), Fault diagnosis of ball bearings using continuous wavelet transform, Applied Soft Computing, 11, 2, 2300-2312.

11. Kareem B. (2015), Evaluation of failures in mechanical crankshafts of automobile based on expert opinion, Case Studies in Engineering Failure Analysis, 3, 25-33.

12. Lazzerini B., Volpi S.L. (2013), Classifier ensembles to improve the robustness to noise of bearing fault diagnosis, Pattern Analysis and Applications, 16, 2, $235-251$.
13. Liu X., Leimbach K., Hartmann D., Höffer R. (2012), Signal analysis using wavelets for structural damage detection applied to wind energy converters, [in:] Proceedings of the 14th International Conference on Computing in Civil and Building Engineering, Moscow, Russia, 27-29 June.

14. Mehdizadeh M., Khodabakhshi F. (2014), An investigation into failure analysis of interfering part of a steam turbine journal bearing, Case Studies in Engineering Failure Analysis, 2, 2, 61-68.

15. Narendiranath Babu T., Manvel Raj T., LaKShmanan T. (2015), A Review on Application of Dynamic Parameters of Journal Bearing for Vibration and Condition Monitoring, Journal of Mechanics, 31, 4, 391-416.

16. Percival D.B., Walden A.T. (2000), Wavelet methods for time series analysis (Cambridge Series in Statistical and Probabilistic Mathematics), Cambridge University Press.

17. Piotrowski L., Augustyniak B., Chmielewski M. (2010), On the possibility of application of the magnetoacoustic emission intensity measurements for the diagnosis of thick-walled objects in the industrial environment, Measurements Science and Technology, 21, $1,1-8$.

18. Rafiee J., Rafiee M.A., Tse P.W. (2010), Application of mother wavelet functions for automatic gear and bearing fault diagnosis, Expert Systems with Applications, 37, 6, 4568-4579.

19. Srinivasan P., Jamieson L.H. (1998), High quality audio compression using an adaptive wavelet packet decomposition and psychoacoustic modelling, IEEE Transaction on Signal Processing, 46, 4, 1085-1093, doi: $10.1109 / 78.668558$.

20. Tianrui Z., Zhenyu W., Tianbiao Y., Wanshan W., HAIFENG Z. (2013), Research on fault diagnosis for TBM based on wavelet packet transforms and BP neural, IEEE 3rd International Advance Computing Conference (IACC), pp. 677-681.

21. Tse P.W., Yang W-X., Tam H.Y. (2004), Machine fault diagnosis through an effective exact wavelet analysis, Journal of Sound and Vibration, 277, 4-5, 10051024.

22. Walnut D.F. (2004), An introduction to wavelet analysis, Springer, Boston.

23. WANG H., Chen P. (2011), Intelligent diagnosis method for rolling element bearing faults using possibility theory and neural network, Computers \& Industrial Engineering, 60, 4, 511-518.

24. Xu W., Ogrodnik P.J., Goodwin M.J., BanCroft G.A. (2009), The Stability Analysis of Hydrodynamic Journal Bearings Allowing for Manufacturing Tolerances. Part I. Effect Analysis of Manufacturing Tolerances by Taguchi Method, Proceedings of International Conference on Measuring Technology and Mechatronics Automation, Hunan, Vol. 2, pp. 164-167. 